\title{
POLARIMETRIC AND PHOTOMETRIC INVESTIGATIONS OF LACERTIDS
}

\author{
V.A. Hagen-Thorn, V.A. Yacovleva \\ Department of Astronomy, Leningrad State University \\ Bibliotechnaja Pl. 2, \\ Leningrad, Petrodvoretz, 198904 \\ USSR
}

\section{ABSTRACT}

At the Astronomical Observatory of Leningrad State University two observational programs of investigation of lacertids - photographic photometry and photoelectric polarimetry - are in progress for many years. Some results for $0 \mathrm{~J} 287$ and BL Lac are of interest: (1) existence of preferable direction of polarization, (2) correlations between the brightness and polarization parameters, and (3) high degree of polarization $(250 \%)$ of individual sources responsible for variability.

The problem of activity of extragalactic objects is one of the main problems of current astrophysics. During the last 30 years, since the time when it had been formulated, an appreciable progress in solving this problem has been achieved but many questions still need elucidation. In particular, it is not quite clear what is the central source responsible for energy output and what are the concrete mechanisms of this output.

Many extragalactic objects demonstrate the activity of different levels but lacertids are the most active. They show high variable polarization and strong rapid photometric variability in optical region. Lacertids are the most suitable objects for investigating the properties of central source since it radiates mostly in continuum. In fact, optical spectra of lacertids are almost continuous, thus it is not necessary to correct data for emission and absorption details.

In this paper we discuss the results of polarimetric and photometric investigations of some lacertids; their observations are in progress for about 15 years at the Astronomical Observatory of Leningrad State University and we have long homogeneous sets of observations.

Photographic monitoring of lacertids for the purpose of constructing their light curves is carried out by $45-\mathrm{cm}$ telescope; photoelectric polarimetry and photometry - by $48-\mathrm{cm}$ reflector. The latter is small and duration of one polarimetric observation is about $1.0-1.5$ hours. Comparison of our results with those received by large telescopes show good agreement [1]. 
We start with the discussion of the question concerning presence or absence of preferable direction of polarization in lacertids. By this feature Angel and Stockman [2] divided all lacertids in two groups but we feel that the conclusion about absence of preferable direction for some lacertids arise from the lack of observational data. For example in the case of BL Lac in [2] it is stated that this object has "no tendency for preferable angle". This conclusion is based on sparse observational data. Our numerous observations show undoubtedly that preferable direction $\left(\theta_{0} \approx 20^{\circ}\right)$ exists. This fact is illustrated by Fig. 1, where the dashed line gives the distribution of observed angles from our data only (316 points), the solid one - from all the available data (378 points). In fact, all lacertids studied in our program (0J 287 [3], Mrk 421 [4], B2 1418+54 and OI 090.4 [5], BL Lac [6]) have preferable direction of polarization.

We are concerned with the second important question about the existence of correlation in polarization and brightness variability. We investigated this question in details for $0 \mathrm{~J} 287$ [3]. Correlation has been found and for its explanation the existence of constantly acting polarized source in this object was proposed (it is its direction of polarization that is the preferable direction). The sources with other directions are added to this source. At low brightness then only directions close to the preferable one must exist. Fig. 2 shows that this is the case. For BL Lac the situation is similar but less obvious (Fig. 3). Conception about the existence of constantly acting polarized source in lacertids proposed by us in [3] has been supported in $[7,8]$.

Clearing up of the origin of variable sources is of great importance. In [9] we have proposed the method of extracting of the sources of polarized radiation. The application of this method to $0 \mathrm{~J} 287$ and BL Lac shows that the polarization degrees in some sources are as high as $50 \%$ - 60\%. Such high polarization can't be achieved in the case of electron scattering [10] and synchrotron radiation is the only possible explanation. Colorimetric data also give arguments for synchrotron origin of variable sources.

Since the radiation of polarized sources is synchrotron in nature, the existence of preferable direction of polarization (determined by magnetic field direction) is the indication of the existence of stable magnetic fields in lacertids. In [7] constantly acting polarized source is identified with jet. We agree to this conclusion.

\section{REFERENCES}

1. V.A. Hagen-Thorn, S.G. Marchenko, V.A. Yacovleva - Astron. J. of USSR, 61, 925, 1984.

2. J.R.P. Angel, H.S. Stockman - Ann. Rev. Astron. Astrophys., 18, 321, 1980.

3. V.A. Hagen-Thorn - Astrophys. Sp. Sci., 73, 263, 1980.

4. V.A. Hagen-Thorn, S.G. Marchenko, R.I. Smekhacheva, V.A. Yacovleva Astrofizika, 19, 199, 1983.

5. S.G. Marchenko - Astrofizika, 22, 15, 1985.

6. V.A. Hagen-Thorn, S.G. Marchenko, V.A. Yacovleva - Astrofizika, 22, 5, 1985.

7. C. Brindle, J.H. Hough, J.A. Baily et al. - Mon. Not. RAS, 214, 619 1985. 
8. M.L. Sitko, G.D. Schmidt, W.A. Stein - Astrophys. J. Suppl., 59, 323, 1985.

9. V.A. Hagen-Thorn - Transactions Astron. Obs. Leningrad State Univ., $36,20,1981$.

10. V.M. Loskutov, V.V. Sobolev - Astrofizika, 23, 307, 1985.

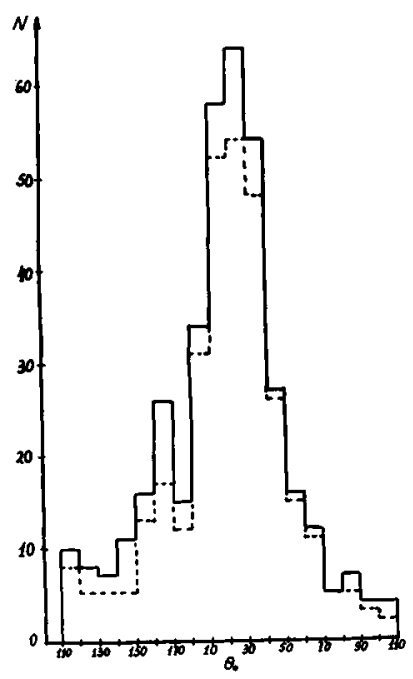

Fig. 1. The distribution of observed polarization angles for BL Lac (dashed line corresponds to our data, solid line - to all available data).

Fig. 3. The same as on Fig. 2 for BL Lac (dots - our data, crosses - data taken from literature).

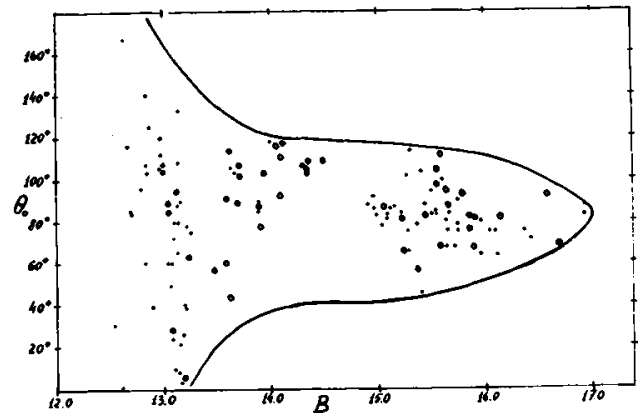

Fig. 2. Comparison betiveen polarisation angle and brightness for OJ 287 (circles correspond to our data, dots - to data taken from literature).

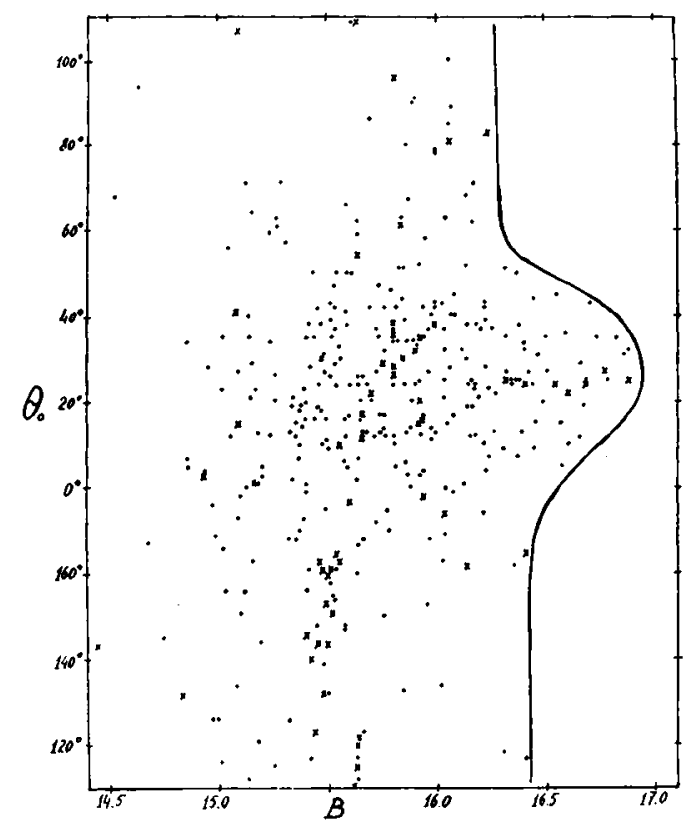

